\title{
A Fixed Point Approach to Superstability of Generalized Derivations on Non-Archimedean Banach Algebras
}

\author{
M. Eshaghi Gordji, ${ }^{1,2}$ M. B. Ghaemi, ${ }^{3}$ and Badrkhan Alizadeh ${ }^{4}$ \\ ${ }^{1}$ Department of Mathematics, Semnan University, P.O. Box 35195-363, Semnan, Iran \\ ${ }^{2}$ Center of Excellence in Nonlinear Analysis and Applications (CENAA), Semnan University, \\ Semnan, Iran \\ ${ }^{3}$ Department of Mathematics, Iran University of Science and Technology, Narmak, Tehran, Iran \\ ${ }^{4}$ The Holy Prophet Higher Education Complex, Tabriz College of Technology, P.O. Box 51745-135, \\ Tabriz, Iran \\ Correspondence should be addressed to M. Eshaghi Gordji, madjid.eshaghi@gmail.com
}

Received 27 February 2011; Revised 6 July 2011; Accepted 18 July 2011

Academic Editor: Ngai-Ching Wong

Copyright (C) 2011 M. Eshaghi Gordji et al. This is an open access article distributed under the Creative Commons Attribution License, which permits unrestricted use, distribution, and reproduction in any medium, provided the original work is properly cited.

We investigate the superstability of generalized derivations in non-Archimedean algebras by using a version of fixed point theorem via Cauchy functional equation.

\section{Introduction}

A functional equation $(\xi)$ is superstable if every approximately solution of $(\xi)$ is an exact solution of it.

The stability of functional equations was first introduced by Ulam [1] during his talk before a Mathematical Colloquium at the University of Wisconsin in 1940.

Given a metric group $G(\cdot, \rho)$, a number $\varepsilon>0$, and a mapping $f: G \rightarrow G$ which satisfies the inequality $\rho(f(x \cdot y), f(x) \cdot f(y)) \leq \varepsilon$ for all $x, y$ in $G$, does there exist an automorphism $a$ of $G$ and a constant $k>0$, depending only on $G$ such that $\rho(a(x), f(x)) \leq k \varepsilon$ for all $x \in G$ ?

If the answer is affirmative, we would call the equation $a(x \cdot y)=a(x) \cdot a(y)$ of automorphism is stable. In 1941, Hyers [2] gave a first affirmative answer to the question of Ulam for Banach spaces. In 1978, Rassias [3] generalized the theorem of Hyers by considering the stability problem with unbounded Cauchy differences $\|f(x+y)-f(x)-f(y)\| \leq$ $\epsilon\left(\|x\|^{p}+\|y\|^{p}\right),(\epsilon>0, p \in[0,1))$. In 1991, Gajda [4] answered the question for the case 
$p>1$, which was raised by Rassias. This new concept is known as Hyers-Ulam-Rassias or generalized Hyers-Ulam stability of functional equations [5, 6].

In 1992, Găvruţa [7] generalized the Th. M. Rassias Theorem as follows.

Suppose that $(G,+)$ is an ablian group, $X$ is a Banach space $\varphi: G \times G \rightarrow[0, \infty)$ which satisfies

$$
\tilde{\varphi}(x, y)=\frac{1}{2} \sum_{n=0}^{\infty} 2^{-n} \varphi\left(2^{n} x, 2^{n} y\right)<\infty
$$

for all $x, y \in G$. If $f: G \rightarrow X$ is a mapping with

$$
\|f(x+y)-f(x)-f(y)\| \leq \varphi(x, y)
$$

for all $x, y \in G$, then there exists a unique mapping $T: G \rightarrow X$ such that $T(x+y)=T(x)+T(y)$ and $\|f(x)-T(x)\| \leq \tilde{\varphi}(x, x)$ for all $x, y \in G$.

In 1949, Bourgin [8] proved the following result, which is sometimes called the superstability of ring homomorphisms: suppose that $A$ and $B$ are Banach algebras with unit. If $f: A \rightarrow B$ is a surjective mapping such that

$$
\begin{gathered}
\|f(x+y)-f(x)-f(y)\| \leq \epsilon, \\
\|f(x y)-f(x) f(y)\| \leq \delta,
\end{gathered}
$$

for some $\epsilon \geq 0, \delta \geq 0$ and for all $x, y \in A$, then $f$ is a ring homomorphism.

Badora [9] and Miura et al. [10] proved the Ulam-Hyers stability and the Isac and Rassias-type stability of derivations [11] (see also [12, 13]); Savadkouhi et al. [14] have contributed works regarding the stability of ternary Jordan derivations. Jung and Chang [15] investigated the stability and superstability of higher derivations on rings. Recently, AnsariPiri and Anjidani [16] discussed the superstability of generalized derivations on Banach algebras. In this paper, we investigate the superstability of generalized derivations on nonArchimedean Banach algebras by using the fixed point methods.

\section{Preliminaries}

In 1897, Hensel [17] has introduced a normed space which does not have the Archimedean property. It turned out that non-Archimedean spaces have many nice applications $[18,19]$.

A non-Archimedean field is a field $\mathbb{K}$ equipped with a function (valuation) $|\cdot|$ from $\mathbb{K}$ into $[0, \infty)$ such that $|r|=0$ if and only if $r=0,|r s|=|r||s|$, and $|r+s| \leq \max \{|r|,|s|\}$ for all $r, s \in \mathbb{K}($ see $[20,21])$.

Definition 2.1. Let $X$ be a vector space over a scalar field $\mathbb{K}$ with a non-Archimedean nontrivial valuation $|\cdot|$. A function $\|\cdot\|: X \rightarrow \mathbb{R}$ is a non-Archimedean norm (valuation) if it satisfies the following conditions:

$\left(\mathrm{NA}_{1}\right)\|x\|=0$ if and only if $x=0$,

$\left(\mathrm{NA}_{2}\right)\|r x\|=|r|\|x\|$ for all $r \in \mathbb{K}$ and $x \in X$,

$\left(\mathrm{NA}_{3}\right)\|x+y\| \leq \max \{\|x\|,\|y\|\}$ for all $x, y \in X$ (the strong triangle inequality). 
A sequence $\left\{x_{m}\right\}$ in a non-Archimedean space is Cauchy if and only if $\left\{x_{m+1}-x_{m}\right\}$ converges to zero. By a complete non-Archimedean space, we mean one in which every Cauchy sequence is convergent. A non-Archimedean normed algebra is a non-Archimedean normed space $A$ with a linear associative multiplication, satisfying $\|x y\| \leq\|x\|\|y\|$ for all $x, y \in A$. A non-Archimedean complete normed algebra is called a non-Archimedean Banach algebra (see [22]).

Example 2.2. Let $p$ be a prime number. For any nonzero rational number $x=(a / b) p^{n_{x}}$ such that $a$ and $b$ are integers not divisible by $p$, define the $p$-adic absolute value $|x|_{p}:=p^{-n_{x}}$. Then, $|\cdot|$ is a non-Archimedean norm on $\mathbb{Q}$. The completion of $\mathbb{Q}$ with respect to $|\cdot|$ is denoted by $\mathbb{Q}_{p}$ which is called the $p$-adic number field.

Definition 2.3. Let $X$ be a nonempty set and $d: X \times X \rightarrow[0, \infty]$ satisfy the following properties:

$\left(\mathrm{D}_{1}\right) d(x, y)=0$ if and only if $x=y$,

$\left(\mathrm{D}_{2}\right) d(x, y)=d(y, x)$ (symmetry),

$\left(\mathrm{D}_{3}\right) d(x, z) \leq \max \{d(x, y), d(y, z)\}$ (strong triangle in equality),

for all $x, y, z \in X$. Then, $(X, d)$ is called a non-Archimedean generalized metric space. $(X, d)$ is called complete if every $d$-Cauchy sequence in $X$ is $d$-convergent.

Definition 2.4. Let $A$ be a non-Archimedean algebra. An additive mapping $D: A \rightarrow A$ is said to be a ring derivation if $D(x y)=D(x) y+x D(y)$ for all $x, y \in A$. An additive mapping $H$ : $A \rightarrow A$ is said to be a generalized ring derivation if there exists a ring derivation $D: A \rightarrow A$ such that

$$
H(x y)=x H(y)+D(x) y,
$$

for all $x, y \in A$.

We need the following fixed point theorem (see $[23,24])$.

Theorem 2.5 (non-Archimedean alternative Contraction Principle). Suppose that $(X, d)$ is a non-Archimedean generalized complete metric space and $\Lambda: X \rightarrow X$ is a strictly contractive mapping; that is,

$$
d(\Lambda x, \Lambda y) \leq L d(x, y), \quad(x, y \in X)
$$

for some $L<1$. If there exists a nonnegative integer $k$ such that $d\left(\Lambda^{k+1} x, \Lambda^{k} x\right)<\infty$ for some $x \in X$, then the followings are true:

(a) the sequence $\left\{\Lambda^{n} x\right\}$ converges to a fixed point $x^{*}$ of $\Lambda$,

(b) $x^{*}$ is a unique fixed point of $\Lambda$ in

$$
X^{*}=\left\{y \in X \mid d\left(\Lambda^{k} x, y\right)<\infty\right\},
$$

(c) if $y \in X^{*}$, then

$$
d\left(y, x^{*}\right) \leq d(\Lambda y, y)
$$




\section{Non-Archimedean Superstability of Generalized Derivations}

Hereafter, we will assume that $A$ is a non-Archimedean Banach algebra with unit over a nonArchimedean field $\mathbb{K}$.

Theorem 3.1. Let $\varphi: A \times A \rightarrow[0, \infty)$ be a function. Suppose that $f, g: A \rightarrow A$ are mappings such that $g$ is additive and

$$
\begin{aligned}
& \|f(x+y)-f(x)-f(y)\| \leq \varphi(x, y), \\
& \|f(x y)-x f(y)-g(x) y\| \leq \varphi(x, y),
\end{aligned}
$$

for all $x, y \in A$. If there exists a natural number $k \in \mathbb{K}$ and $0<L<1$,

$$
|k|^{-1} \varphi(k x, k y),|k|^{-1} \varphi(k x, y),|k|^{-1} \varphi(x, k y) \leq L \varphi(x, y),
$$

for all $x, y \in A$. Then, $f$ is a generalized ring derivation and $g$ is a ring derivation.

Proof. By induction on $i$, we prove that

$$
\|f(i x)-i f(x)\| \leq \max \{\varphi(0,0), \varphi(x, x), \varphi(2 x, x), \ldots, \varphi((i-1) x, x)\},
$$

for all $x \in A$ and $i \geq 2$. Let $x=y$ in (3.1). Then,

$$
\|f(2 x)-2 f(x)\| \leq \max \{\varphi(0,0), \varphi(x, x)\}, \quad n \in \mathbb{N}_{0}, x \in A .
$$

This proves (3.4) for $i=2$. Let (3.4) holds for $i=1,2, \ldots, j$. Replacing $x$ by $j x$ and $y$ by $x$ in (3.1) for each $n \in \mathbb{N}_{0}$, and for all $x \in A$, we get

$$
\|f((j+1) x)-f(j x)-f(x)\| \leq \max \{\varphi(0,0), \varphi(j x, x)\} .
$$

Since

$$
\begin{aligned}
& f((j+1) x)-f(j x)-f(x) \\
& \quad=f((j+1) x)-(j+1) f(x)+(j+1) f(x)-f(j x)-f(x) \\
& \quad=f((j+1) x)-(j+1) f(x)+j f(x)-f(j x),
\end{aligned}
$$

for all $x \in A$, it follows from induction hypothesis and (3.6) that

$$
\begin{aligned}
& \|f((j+1) x)-(j+1) f(x)\| \\
& \quad \leq \max \{\|f((j+1) x)-f(j x)-f(x)\|,\|j f(x)-f(j x)\|\} \\
& \quad \leq \max \{\varphi(0,0), \varphi(x, x), \varphi(2 x, x), \ldots, \varphi((j) x, x)\},
\end{aligned}
$$


for all $x \in A$. This proves (3.4) for all $i \geq 2$. In particular,

$$
\|f(k x)-k f(x)\| \leq \psi(x)
$$

for all $x \in A$ where

$$
\psi(x)=\max \{\varphi(0,0), \varphi(x, x), \varphi(2 x, x), \ldots, \varphi((k-1) x, x)\} \quad(x \in A) .
$$

Let $X$ be the set of all functions $r: A \rightarrow A$. We define $d: X \times X \rightarrow[0, \infty]$ as follows:

$$
d(r, s)=\inf \{\alpha>0:\|r(x)-s(x)\| \leq \alpha \psi(x) \forall x \in A\} .
$$

It is easy to see that $d$ defines a generalized complete metric on $X$. Define $J: X \rightarrow X$ by $J(r)(x)=k^{-1} r(k x)$. Then, $J$ is strictly contractive on $X$, in fact, if

$$
\|r(x)-s(x)\| \leq \alpha \psi(x), \quad(x \in A)
$$

then by (3.3),

$$
\|J(r)(x)-J(s)(x)\|=|k|^{-1}\|r(k x)-s(k x)\| \leq \alpha|k|^{-1} \psi(k x) \leq L \alpha \psi(x), \quad(x \in A) .
$$

It follows that

$$
d(J(r), J(s)) \leq L d(r, s) \quad(r, s \in X) .
$$

Hence, $J$ is a strictly contractive mapping with Lipschitz constant L. By (3.9),

$$
\begin{gathered}
\|(J f)(x)-f(x)\|=\left\|k^{-1} f(k x)-f(x)\right\|, \\
|k|^{-1}\|f(k x)-k f(x)\| \leq|k|^{-1} \psi(x) \quad(x \in A) .
\end{gathered}
$$

This means that $d(J(f), f) \leq 1 /|k|$. By Theorem 2.5, $J$ has a unique fixed point $h: A \rightarrow A$ in the set

$$
U=\{r \in X: d(r, J(f))<\infty\}
$$

and for each $x \in A$,

$$
h(x)=\lim _{m \rightarrow \infty} J^{m}(f(x))=\lim k^{-m} f\left(k^{m} x\right) .
$$


Therefore,

$$
\begin{aligned}
& \|h(x+y)-h(x)-h(y)\| \\
& \quad=\lim _{m \rightarrow \infty}|k|^{-m}\left\|f\left(k^{m}(x+y)\right)-f\left(k^{m} x\right)-f\left(k^{m} y\right)\right\| \\
& \quad \leq \lim _{m \rightarrow \infty}|k|^{-m} \max \left\{\varphi(0,0), \varphi\left(k^{n} x, k^{n} y\right)\right\} \\
& \quad \leq \lim _{m \rightarrow \infty} L^{m} \varphi(x, y)=0,
\end{aligned}
$$

for all $x, y \in A$. This shows that $h$ is additive.

Replacing $x$ by $k^{n} x$ in (3.2) to get

$$
\left\|f\left(k^{n} x y\right)-k^{n} x f(y)-g\left(k^{n} x\right) y\right\| \leq \varphi\left(k^{n} x, y\right),
$$

and so

$$
\left\|\frac{f\left(k^{n} x y\right)}{k^{n}}-x f(y)-\frac{g\left(k^{n} x\right)}{k^{n}} y\right\| \leq \frac{1}{|k|^{n}} \varphi\left(k^{n} x, y\right) \leq L^{n} \varphi(x, y),
$$

for all $x, y \in A$ and all $n \in \mathbb{N}$. By taking $n \rightarrow \infty$, we have

$$
h(x y)=x f(y)+\lim _{n \rightarrow \infty} \frac{g\left(k^{n} x\right)}{k^{n}} y
$$

for all $x, y \in A$.

Fix $m \in \mathbb{N}$. By (3.21), we have

$$
\begin{aligned}
x f\left(k^{m} y\right) & =h\left(k^{m} x y\right)-\lim _{n \rightarrow \infty}\left(\frac{g\left(k^{n} x\right)}{k^{n}}\left(k^{m} y\right)\right) \\
& =k^{m} x f(y)+\lim _{n \rightarrow \infty}\left(\frac{g\left(k^{n} k^{m} x\right)}{k^{n}} y\right)-k^{m} \lim _{n \rightarrow \infty}\left(\frac{g\left(k^{n} x\right)}{k^{n}} y\right) \\
& =k^{m} x f(y)+k^{m} \lim _{n \rightarrow \infty}\left(\frac{g\left(k^{n+m} x\right)}{k^{n+m}} y\right)-k^{m} \lim _{n \rightarrow \infty}\left(\frac{g\left(k^{n} x\right)}{k^{n}} y\right) \\
& =k^{m} x f(y),
\end{aligned}
$$

for all $x, y \in A$. Then, $x f(y)=x\left(f\left(k^{m} y\right) / k^{m}\right)$ for all $x, y \in A$ and each $m \in \mathbb{N}$, and so by taking $m \rightarrow \infty$, we have $x f(y)=x h(y)$. Now, we obtain $h=f$, since $A$ is with unit. Replacing $y$ by $k^{n} y$ in (3.2), we obtain

$$
\left\|f\left(k^{n}(x y)\right)-x f\left(k^{n} y\right)-k^{n} g(x) y\right\| \leq \varphi\left(x, k^{n} y\right)
$$


and hence,

$$
\left\|\frac{f\left(k^{n} x y\right)}{k^{n}}-x \frac{f\left(k^{n} y\right)}{k^{n}}-g(x) y\right\| \leq \frac{1}{|k|^{n}} \varphi\left(x, k^{n} y\right) \leq L^{n} \varphi(x, y),
$$

for all $x, y \in A$ and each $n \in \mathbb{N}$. Letting $n$ tends to infinite, we have

$$
f(x y)=x f(y)+g(x) y
$$

Now, we show that $g$ is a ring derivation. By (3.25), we get

$$
\begin{aligned}
g(x y) z & =f(x y z)-x y f(z) \\
& =x f(y z)+g(x) y z-x y f(z) \\
& =(x g(y)+g(x) y) z
\end{aligned}
$$

for all $x, y, z \in A$. Therefore, we have $g(x y)=x g(y)+g(x) y$.

The proof of following theorem is similar to that in Theorem 3.1, hence it is omitted.

Theorem 3.2. Let $\varphi: A \times A \rightarrow[0, \infty)$ be a function. Suppose that $f, g: A \rightarrow A$ are mappings such that $g$ is additive and

$$
\begin{aligned}
& \|f(x+y)-f(x)-f(y)\| \leq \varphi(x, y) \\
& \|f(x y)-x f(y)-g(x) y\| \leq \varphi(x, y)
\end{aligned}
$$

for all $x, y \in A$. If there exists a natural number $k \in \mathbb{K}$ and $0<L<1$,

$$
|k| \varphi\left(k^{-1} x, k^{-1} y\right),|k| \varphi\left(k^{-1} x, y\right),|k| \varphi\left(x, k^{-1} y\right) \leq L \varphi(x, y)
$$

for all $x, y \in A$. Then, $f$ is a generalized ring derivation and $g$ is a ring derivation.

The following results are immediate corollaries of Theorems 3.1 and 3.2 and Example 2.3.

Corollary 3.3. Let $A$ be a non-Archimedean Banach algebra over $\mathbb{Q}_{p}, \varepsilon>0$, and $p_{1}, p_{2} \in(1, \infty)$. Suppose that $f, g: A \rightarrow$ A are mappings such that $g$ is additive and

$$
\begin{aligned}
& \|f(x+y)-f(x)-f(y)\| \leq \varepsilon\left(\|x\|^{p_{1}}\|y\|^{p_{2}}\right), \\
& \|f(x y)-x f(y)-g(x) y\| \leq \varepsilon\left(\|x\|^{p_{1}}\|y\|^{p_{2}}\right),
\end{aligned}
$$

for all $x, y \in A$. Then, $f$ is a generalized ring derivation and $g$ is a ring derivation. 
Corollary 3.4. Let $A$ be a non-Archimedean Banach algebra over $\mathbb{Q}_{p}, \varepsilon>0$ and $p_{1}, p_{2}, p_{1}+p_{2} \in$ $(-\infty, 1)$. Suppose that $f, g: A \rightarrow A$ are mappings such that $g$ is additive and

$$
\begin{aligned}
& \|f(x+y)-f(x)-f(y)\| \leq \varepsilon\left(\|x\|^{p_{1}}\|y\|^{p_{2}}\right), \\
& \|f(x y)-x f(y)-g(x) y\| \leq \varepsilon\left(\|x\|^{p_{1}}\|y\|^{p_{2}}\right),
\end{aligned}
$$

for all $x, y \in A$. Then, $f$ is a generalized ring derivation and $g$ is a ring derivation.

\section{References}

[1] S. M. Ulam, Problems in Modern Mathematics, chapter 6, Wiley, New York, NY, USA, science edition, 1940.

[2] D. H. Hyers, "On the stability of the linear functional equation," Proceedings of the National Academy of Sciences of the United States of America, vol. 27, pp. 222-224, 1941.

[3] T. M. Rassias, "On the stability of the linear mapping in Banach spaces," Proceedings of the American Mathematical Society, vol. 72, no. 2, pp. 297-300, 1978.

[4] Z. Gajda, "On stability of additive mappings," International Journal of Mathematics and Mathematical Sciences, vol. 14, no. 3, pp. 431-434, 1991.

[5] M. E. Gordji and H. Khodaei, Stability of Functional Equations, LAP LAMBERT, 2010.

[6] D. H. Hyers, G. Isac, and T. M. Rassias, Stability of Functional Equations in Several Variables, Birkhaěr, Basel, Switzerland, 1998.

[7] P. Găvruţa, "A generalization of the Hyers-Ulam-Rassias stability of approximately additive mappings," Journal of Mathematical Analysis and Applications, vol. 184, no. 3, pp. 431-436, 1994.

[8] D. G. Bourgin, "Approximately isometric and multiplicative transformations on continuous function rings," Duke Mathematical Journal, vol. 16, pp. 385-397, 1949.

[9] R. Badora, "On approximate derivations," Mathematical Inequalities E Applications, vol. 9, no. 1, pp. 167-173, 2006.

[10] T. Miura, G. Hirasawa, and S. E. Takahasi, "A perturbation of ring derivations on Banach algebras," Journal of Mathematical Analysis and Applications, vol. 319, no. 2, pp. 522-530, 2006.

[11] G. Isac and T. M. Rassias, "On the Hyers-Ulam stability of $\psi$-additive mappings," Journal of Approximation Theory, vol. 72, no. 2, pp. 131-137, 1993.

[12] M. E. Gordji, "Nearly ring homomorphisms and nearly ring derivations on non-Archimedean Banach algebras," Abstract and Applied Analysis, vol. 2010, Article ID 393247, 12 pages, 2010.

[13] M. E. Gordji and Z. Alizadeh, "Stability and superstability of ring homomorphisms on nonArchimedean Banach algebras," Abstract and Applied Analysis, vol. 2011, Article ID 123656, 10 pages, 2011.

[14] M. B. Savadkouhi, M. E. Gordji, J. M. Rassias, and N. Ghobadipour, "Approximate ternary Jordan derivations on Banach ternary algebras," Journal of Mathematical Physics, vol. 50, no. 4, Article ID 042303, 9 pages, 2009.

[15] Y.-S. Jung and I.-S. Chang, “On approximately higher ring derivations," Journal of Mathematical Analysis and Applications, vol. 343, no. 2, pp. 636-643, 2008.

[16] E. Ansari-Piri and E. Anjidani, "Superstability of generalized derivations," Journal of Inequalities and Applications, vol. 2010, Article ID 740156, 13 pages, 2010.

[17] K. Hensel, “Uber eine neue Begrundung der theorie der algebraischen Zahlen," Jahresbericht der Deutschen Mathematiker-Vereinigung, vol. 6, pp. 83-88, 1897.

[18] A. Khrennikov, Non-Archimedean Analysis: Quantum Paradoxes, Dynamical Systems and Biological Models, vol. 427 of Mathematics and its Applications, Kluwer Academic Publishers, Dordrecht, The Netherlands, 1997.

[19] A. C. M. van Rooij, Non-Archimedean Functional Analysis, vol. 51 of Monographs and Textbooks in Pure and Applied Math., Marcel Dekker, New York, NY, USA, 1978.

[20] L. M. Arriola and W. A. Beyer, "Stability of the Cauchy functional equation over p-adic fields," Real Analysis Exchange, vol. 31, no. 1, pp. 125-132, 2005-2006.

[21] M. S. Moslehian and T. M. Rassias, "Stability of functional equations in non-Archimedean spaces," Applicable Analysis and Discrete Mathematics, vol. 1, no. 2, pp. 325-334, 2007. 
[22] J. A. Alvarez, "C ${ }^{*}$-algebras of operators in non-Archimedean Hilbert spaces," Commentationes Mathematicae Universitatis Carolinae, vol. 33, no. 4, pp. 573-580, 1992.

[23] A. K. Mirmostafaee, "Non-Archimedean stability of the monomial functional equations," Tamsui Oxford Journal of Mathematical Sciences, vol. 26, no. 2, pp. 221-235, 2010.

[24] A. K. Mirmostafaee, "Non-Archimedean stability of quadratic equations," Fixed Point Theory, vol. 11, no. 1, pp. 67-75, 2010. 


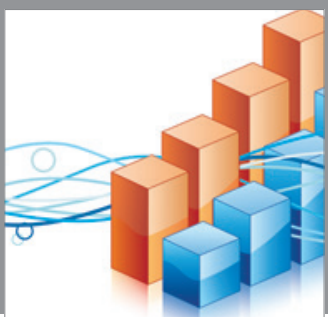

Advances in

Operations Research

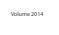

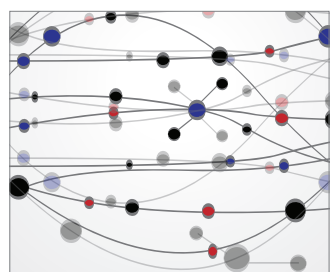

\section{The Scientific} World Journal
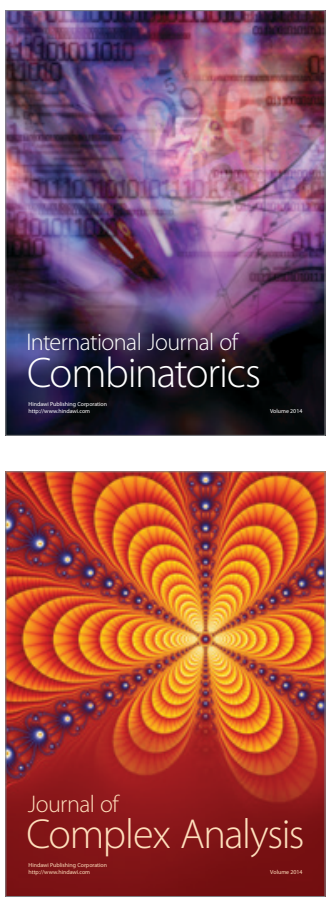

International Journal of

Mathematics and

Mathematical

Sciences
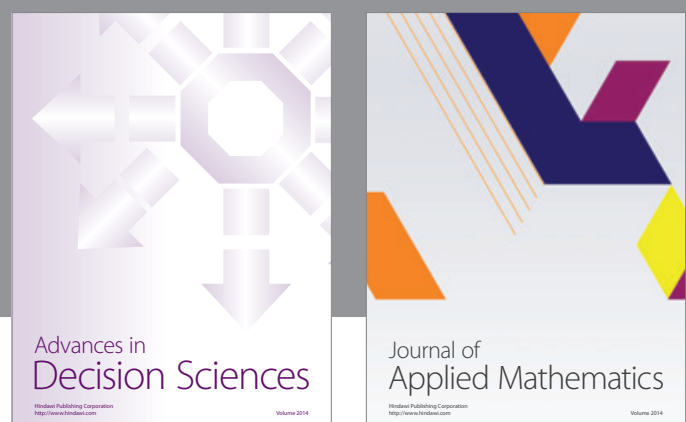

Journal of

Applied Mathematics
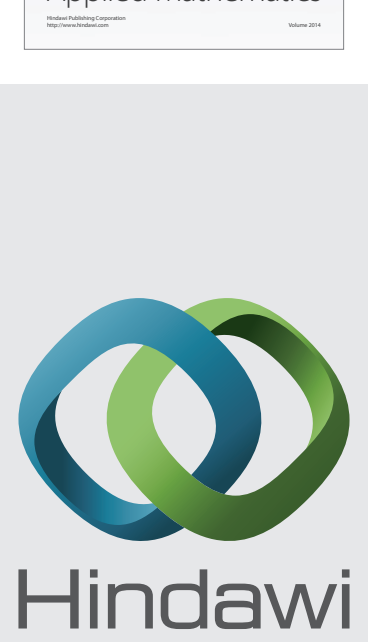

Submit your manuscripts at http://www.hindawi.com
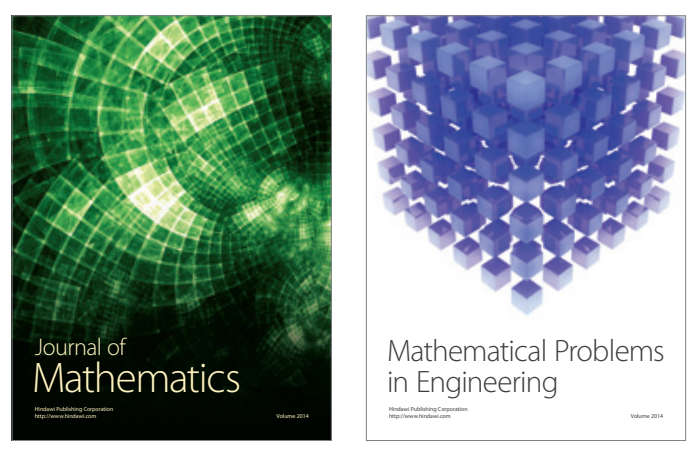

Mathematical Problems in Engineering
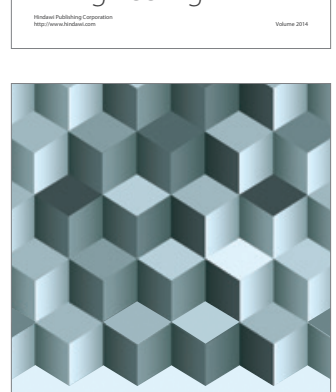

Journal of

Function Spaces
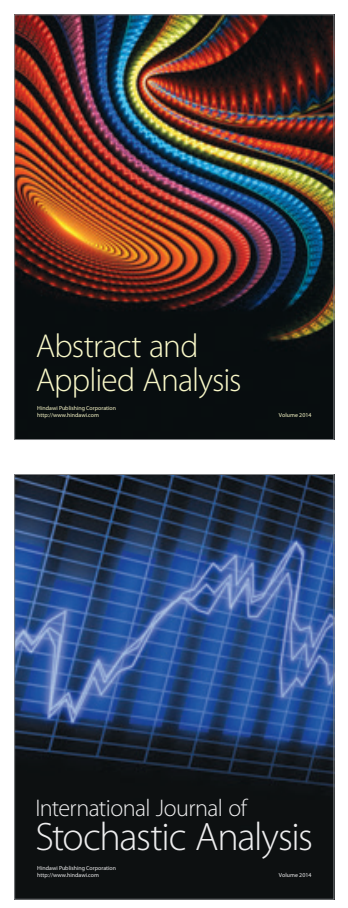

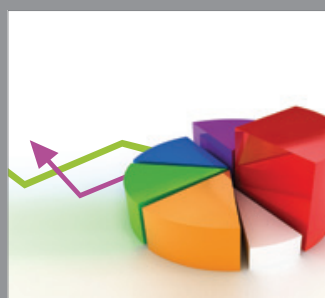

ournal of

Probability and Statistics

Promensencen
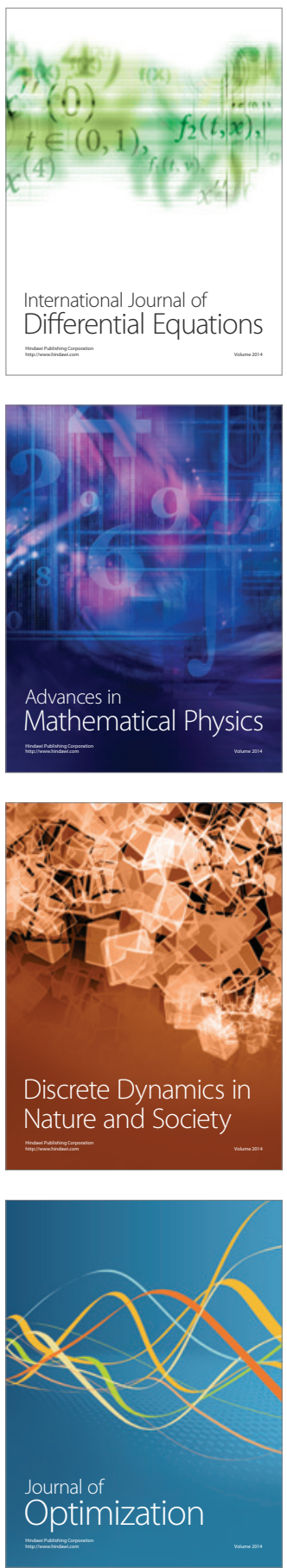